\title{
Are We Learning as Much as Possible from Spaceflight to Better Understand Health and Risks to Health on Earth, as Well as in Space?
}

\author{
David A. Hart1,2,3,4,5
}

\begin{abstract}
${ }^{1}$ Human Performance Laboratory, University of Calgary, Calgary, Canada; ${ }^{2}$ Faculty of Kinesiology, University of Calgary, Calgary, Canada; ${ }^{3}$ Department of Surgery, University of Calgary, Calgary, Canada; ${ }^{4} \mathrm{McCaig}$ Institute for Bone \& Joint Health, University of Calgary, Calgary, Canada; ${ }^{5}$ Alberta Health Services Bone \& Joint Health Strategic Clinical Network, Edmonton, Canada
\end{abstract}

Correspondence to: David A. Hart, Hartd@ucalgary.ca

Keywords: Physiology, Genome, Epigenome, Space Flight, Precision Medicine, Precision Health, Silent Mutations, Accelerated Aging

Received: May 8, 2018 Accepted: June 10, $2018 \quad$ Published: June 13, 2018

Copyright $\odot 2018$ by author and Scientific Research Publishing Inc.

This work is licensed under the Creative Commons Attribution International License (CC BY 4.0).

http://creativecommons.org/licenses/by/4.0/

\section{(c) (i) Open Access}

\section{ABSTRACT}

The objective of this review is to discuss the changes in human biology and physiology that occur when humans, who evolved on Earth for millions of years, now are subjected to space flight for extended periods of time, and how detailing such changes associated with space flight could help better understand risks for loss of health on Earth. Space programs invest heavily in the selection and training of astronauts. They also are investing in maintaining the health of astronauts, both for extensive stays in low earth orbit on ISS, and in preparation for deep space missions in the future. This effort is critical for the success of such missions as the $\mathrm{N}$ is small and the tasks needed to be performed in a hostile environment are complex and demanding. However, space is a unique environment, devoid of many of the "boundary conditions" that shaped human evolution (e.g. 1 g environment, magnetic fields, background radiation, oxygen, water, etc). Therefore, for humans to be successful in space, we need to learn to adapt and minimize the impact of an altered environment on human health. Conversely, we can also learn considerably from this altered environment for life on earth. The question is, are we getting the maximal information from life in space to learn about like on earth? The answer is likely No, and as such, our "Return on Investment" is not as great as it could be. Even though the number of astronauts is not large, what we can learn from them could help shape new questions for research focused on health for those on earth, as well is contribute to "precision health" from the study of astronaut diversity. This 
latter effort would contribute to both the health of astronauts identifying risks, as well as contribute to health on earth via better understanding of the human genome and epigenome, as well as factors contributing to risk for diseases on earth, particularly as individuals age and regulatory systems become altered. Better use of the International Space Station, and similar platforms in the future, could provide critical insights in aging-associated risks for loss of health on Earth, as well as promote new approaches to using precision medicine to overcome threats to health while in space. To achieve this goal will likely require advanced approaches to collecting such information and use of more systems biology, systems physiology approaches to integrate the information.

\section{PREAMBLE}

Life on Earth is very old, has gone through many iterations based on fossil records, is quite diverse at any given point in time, and is very dynamic based on even the relatively modern observations of Darwin [1].

In fact, the Earth itself is very dynamic with regard to both its internal workings (e.g. tectonic plate movements, earthquakes, volcanic eruptions, etc) as well as its relationship with external forces such as the moon, the sun, planets, and seemingly random encounters with objects in space of varying sizes that can impact the status quo of life on Earth.

If one focuses on the internal attributes of the Earth that may have fostered the development of life, it is clear that this planet has characteristics that allowed seemingly stochastic events to lead to self-replicating single cell organisms to arise, likely in the context of the abundant water supply and an evolving atmosphere. Thus, the Earth also has a magnetic field of varying intensity that shields life from the potentially fatal exposure to space radiation, and it is the "right" distance from its star to allow for a zone of temperate extremes to enhance survival of primitive carbon-based species. In addition, the Earth has background radiation from radioactive elements in its crust that could be an enhancer of mutation rates for organisms with a DNA-based self-replicating system, a background which would allow for a rate of adaptation, but not to the extent that would overtly compromise long term survival of primitive organisms.

Thus, life as we know it today is the culmination of survival and adaptation to millions of years of climate change, tectonic plate movement, encounters with space objects which resulted in extinction-level events, evolving predator-prey relationships, and relative stabilization in the context of the background radiation and magnetic field of the Earth (which is however, not uniform in all locations on Earth $[2,3]$ ).

Humanoids, with Homo sapiens the current dominant species have existed for a small "slice" of the continuum of life on the planet. Based on an incomplete fossil record, humanoids have existed for millions of years in one form or another, some forms potentially direct precursors of Homo sapiens, but others likely branches that have not survived for one reason or another. Current theories hypothesize that many steps in the process to Homo sapiens emanated from Africa [4], but this is still a theory, based in part on fossil records. Why humanoids as we know them would have come from one location is not known, but perhaps the constellation of conditions (e.g. background radiation [2], magnetic field variation [3], food sources, reproductive success, etc.) contributed to such a focus of evolution to modern humans.

\section{BACKGROUND}

Modern humans evolved in the context of a $1 \mathrm{~g}$ environment, varying levels of background radiation, a magnetic field of varying location-dependent strength, and an ever changing set of conditions related to weather and the influence of Earth's relationships with external forces. Biologically, modern humans also built on the advantages developed by primitive lifeforms over the eons, retaining those elements in their DNA that contributed to self-replication, survival in the context of the $1 \mathrm{~g}$, magnetic fields and back- 
ground radiation, and abilities to function effectively. Thus, modern humans do share some related genes with fruit flies, worms, fish, and other primates. However, what distinguishes humans is the regulation of such genes, and the adaptation of strategies to "mix and match" genetic elements to enhance survival (e.g. the specific immune system, the olfactory system, enzymes which share exons, etc.). On top of that approach, other mechanisms related to regulation of genes involve epigenetic mechanisms to affect regulation of DNA, as well as hormonal (e.g. estrogens, testosterone, glucocorticoids, neuroendocrine molecules) approaches to affect DNA and expression of genes. In addition, development and application of specific applications to effect DNA repair and enhance replication fidelity, as well as cancer suppressor genes, were likely important to enhance survival and avoid detrimental consequences of living on a planet with a radioactive crust.

Insights into the complexity of regulation resulted from the outcome of the human genome project [5], and continues today. Sequencing the human genome has revealed that a considerable proportion of the DNA does not encode for the 20 - 30,000 genes in the genome. Thus, much sequence is in introns (sequences between coding elements) or in areas which had no known functions a few decades ago. However, it is likely nothing is retained that is without function, so regulatory elements in such areas of the genome are being identified. In addition, the human genome contains several millions of Single Nucleotide Polymorphisms (SNP) both within an individual and between individuals. Such variation may impact coding sequences and thus, the functioning of the expressed protein, or they can exist in promoter regions of the genes, and thus impact the regulation of gene expression. How such diversity contributes to regulation in specific tissues/organs, the aging process, or disease processes remains unknown for the most part. Furthermore, how and why such diversity arose is also not understood-but by implication, this is an active process and if contributing to on-going evolution (a dynamic process, not static-a fact that many do not appreciate), what the impact would be. As the average lifespan of a modern human was likely 40 - 50 years of age until a few centuries ago, such diversity may not have had an impact under those conditions, but influence can be observed now with longer lifespans, more age-related diseases being evident (e.g. dementia, cardiovascular disease, cancer, metabolic disorders). Furthermore, under the 1g, magnetic fields and radiation of Earth, much of such variation may have been "silent" as long as it did not impact survival and reproduction.

Whatever the mechanism(s) responsible for development of such diversity and heterogeneity, it does appear to also provide some survival advantages. As examples, a subset of individuals exposed to the plagues of Europe, the 1918 influenza epidemic, or the HIV pandemic appear to be resistant to life-ending disease, and survive. With humans having paired chromosomes, the heterogeneity of each chromosome provides added diversity to enhance survival. Heterozygosity is therefore, likely the preferred state. Inbreeding in humans leads to a loss of such heterozygosity, and increased risk for homozygosity and abnormal development, maturation, or disease risk. Farmers have known this for centuries regarding healthy herds of pigs, cattle, sheep, etc. Interestingly, many researchers today use completely inbred mice, with such heterogeneity bred out of them for much health related research. In this regard, such mice are a convenient tool, but the results likely do not reflect the real world and only reflects a restricted state, on Earth or in space!

The diversity discussed above has frustrated those who seek to identify genetic risk for diseases by performing "genome-wide" scans of sequences to link to clinically defined diseases. For diseases conveyed by single gene mutations, such approaches can be fruitful. However, most diseases appear to be multi-genic, with contributions from many genes or genomic elements, so it is much harder to track down with clarity which are involved and how. However, some of this effort can be enhanced by investigating ethnic or sex differences in different populations, or family studies where risk is concentrated.

Of importance in this regard is the fact that the "boundary conditions" of the individual on earth has imposed a number of rather unique circumstances on gene regulation, and regulation in general on modern humans. These include dependence on mobility and functioning up-right in a $1 \mathrm{~g}$ environment-working against the gravity of the planet. Secondly, our nervous system is "electrical" in nature, and as such generates nano-biomagnetic fields [6] that must function in the context of the Earth's magnetic 
field, and thus, must exhibit regulatory fidelity against an external field. While the background radiation of the Earth's crust could be an "enhancer" of the mutation rate and thus contribute to a high rate of productive adaptations, it is also a source of detrimental influence on system integrity which is dependent on the fidelity of the DNA-dependence. It is of interest that many cancers occur in early childhood when the system is growing, and in "old" age when an individual is well beyond their reproductive years, so except for some failures in a subset of individuals in young adulthood, the adaptations are for the most part, effective in controlling this risk. Therefore, as long as humans remain on Earth, they are adapted well (but evolution is not over!) and can function effectively for $100+$ years.

\section{SPACE FLIGHT-A UNIQUE OPPORTUNITY TO STUDY HOMO SAPIENS IN AN ALTERED ENVIRONMENT}

The advent of space flight some 60+ years ago has offered the opportunity to study human physiology and psychology in a unique environment, one in which some of the boundary conditions controlling human evolution were removed. This has culminated in the establishment of the International Space Station (ISS), a stable platform to study humans for 6-months to a year, and their responses to microgravity, the effectiveness of countermeasures, living in an isolated confined environment (ICE), an increased exposure to radiation and magnetic storms, and various combinations of the above. In addition, a small subset of astronauts have gone to the moon, beyond some of the Earth's magnetic field and with increased exposure to space radiation. Now plans are underway to travel back to the moon (habitat on the moon, Deep Space Gateway around the moon) and even further to Mars and possibly asteroids, places where risks for radiation exposure and magnetic field alterations are even more prevalent.

A major focus of the life sciences in space thus far, has been on astronaut physical and psychological health, as well as developing effective countermeasures to mitigate the responses to life in space for Homo sapiens. While some studies with cells, animals, plants, and lower life forms (flies, worms, fish) have been performed, the major focus has been around the central theme of astronaut health, with some studies related to life sciences in general.

\section{LESSONS ALREADY LEARNED FROM EARTH AND LOW EARTH ORBIT (LEO)}

Since humans have ventured into space over the past 60+ years, several lessons have been learned regarding responses to microgravity, living in ICE for up to a year in LEO, the effectiveness of counter measures, and the variation in human responses to such changes. The current vehicle of change is the ISS, stranded in LEO, with loss of the $1 \mathrm{~g}$ environment, an altered exposure to radiation and to some extent the geomagnetic field, but living in an artificial magnetic field generated by the equipment of the ISS. Some of the major points/issues identified include:

1) Extensive variability in muscle and bone loss of astronauts on ISS, with exercise counter measures fairly effective for retention of muscle integrity, but less so for bone health, particularly for the lower extremities $[7,8]$. Pharmacologic interventions such as bisphosphonates may be effective regarding bone loss for many astronauts in microgravity, however it remains to be seen whether the $1 / 3 \mathrm{~g}$ of Mars is sufficient to stop or prevent progression of additional bone loss, but likely the $1 / 6 \mathrm{~g}$ of the moon may not be sufficient. Therefore, it remains to be determined if "one size fits all" when it comes to mitigating these risks to components of the mobility system. Better understanding of these areas are likely relevant to age-related development of sarcopenia and osteoporosis on Earth.

2) Human genetic variation likely influences the rate of response to the altered LEO environment, but due to an apparent lack of will to perform pre-flight genome sequencing to identify risk and personalize countermeasures, this remains a gap. However, genetic information can only identify potential risk, and subjecting astronauts to a bed rest protocol pre-flight would potentially identify functional risk for loss of integrity, and subsequent risk for loss of performance. Obviously, developing the ability to better understand genetic risk associated with phenotypic alterations in the unique environment of space could provide insights and direction to improve understanding what some of the myriad of mutations and SNP varia- 
tions might mean to disease development on Earth.

Related to the genetic aspects is also the epigenetic aspects of exposure to space conditions. That is, modifications to the genome which can affect gene regulation and expression is known to occur on earth with aging or exposure to "stressors" during the lifespan [9]. A recent "experiment" with identical twins who are USA astronauts was conducted where one brother remained on Earth and the other brother was on ISS for 1 year. Exposure to ISS condition led to changes in the epigenetic profile compared to the Earth-bound brother, and these changes reversed after a return to Earth [popular press stories]. However, changes to expression of molecules by human cells and known to influence gene regulation have also been reported to be altered on ISS [10]. As a number of epigenetic changes may occur with chronic diseases on Earth [11], and they may impact treatment efficacy, better understanding of the space-related changes and the mechanisms for their reversibility may provide insights to better treat those on Earth with various diseases.

In addition, there are also implications for understanding astronaut health, responsiveness to treatment, and new risks potentially associated with these epigenetic alterations occurring during time in space. Depending on the extent of the changes, whether the changes stabilize to establish a new meta-stable "set point", which genes are affected (and whether they are the same for all astronauts given human diversity), and whether the epigenetic changes occurring in the stable LEO environment would be further altered during deep space missions, while on the moon or Mars, or beyond, likely means the baseline for health while in space is not the same as when on Earth. Thus, spending considerable effort to establish the Earth baseline may not provide the information required to assess what should be considered health while in space, particularly with regard to deep space missions and the altered risk phenotype.

3) Loss of vascular tone can be extensive in astronauts. Based on the work of Hughson $[12,13]$ and others, changes to the integrity of the vascular system, which parallel changes with aging, pose a risk for compromise of performance in the long term, alone or in combination with other threats. Effective counter measures have not yet been identified and effectively implemented to mitigate this threat to performance. More research is required in this area and it is relevant to better understanding of cardiovascular alterations associated with aging on Earth.

4) Exposure to increased levels of radiation, and different types of radiation, can lead to DNA breakage (and there is variation between humans in DNA repair enzymes, [14]), breakage of other molecular bonds (metabolic disturbance, inactivation of antibiotics or other medications with complex ring structures or essential configurations), and risk for cell transformation and cancers. This has not been a realized risk in LEO, possibly due to residual geomagnetic field influence, short duration of flights, or stochastic luck. Cancer risk can be enhanced by the presence of mutated suppressor regulatory genes on Earth. However, these risks have apparently not been assessed in astronauts at the genetic level. Thus, performance could be put at risk by development of a debilitating cancer (treatable or not) during a long duration deep space mission. More research is required in this area and is relevant to cancer risk and development on Earth. Moreover, it is not clear whether such radiation risks in space are also influenced by concurrent alterations leading to microgravity [15].

5) Exposure to an altered geomagnetic field and increased exposure to magnetic storms from the sun appear to be under studied risks to human performance while in space. The human neural system and brain activity is an electrical system which generates magnetic fields that can be assessed by SQUID technology [16]. Thus, the brains of humans developed and function in the context of the geomagnetic field of Earth. While on ISS, humans remain under the influence of part of the geomagnetic field, but they are also living in an artificial magnetic field created by the constant and intermittent running equipment. The only astronauts who have lived for some time outside of much of the geomagnetic field of Earth are those who have gone to the moon. No adverse conditions associated with those missions in this regard could be found in the literature available. However, some publications have implicated a higher risk for cardiovascular events after a return to earth [17, 18], but this is controversial [19]. While strong evidence is lacking as to the basis for such risk (i.e. radiation or magnetic influences), certainly the functioning of the heart is based on electrical stimulation, with an associated biomagnetic field, so this may be within the realm of 
speculation. Of interest is the fact that while there appeared to be increased risk for cardiovascular events $[17,18]$, there were others who did not appear to be affected by this risk. If this risk is confirmed, this latter population could also provide some interesting insights into cardiovascular health for those that are Earth-bound as well.

In addition, astronauts in LEO may be more influenced by exposure to magnetic storms emanating from the sun in a somewhat unpredictable manner. Some reports indicate that exposure to such magnetic storms can impact brain functions and potentially contribute to psychological alterations [20, 21] and thus, performance. However, this area appears to be somewhat understudied. More research is required as this area could provide insights in risks for neural diseases associated with aging on Earth. While in space, such magnetic storm risks to performance may also be further complicated by concurrent radiation risks to the central nervous system [22].

It is interesting that there have been a number of reports regarding the impact of magnetic alterations on Homo sapiens on Earth. For instance Kay [23] reported on the possible relationship between schizophrenia and seasonal variation in geomagnetic storms. Others have used simulated alterations in geomagnetic activity on both the heart [18] and the brain [24] to assess results of correlational studies. Other interest in this area is likely based on controversies regarding whether potential adverse responses to living near power lines is based in the electromagnetic fields generated. How biological diversity of Homo sapiens could contribute to variation in responses to magnetic field alterations on Earth or in space is another area that could be potentially addressed by further study in space that would also have application on Earth. While humans are somewhat protected by the geomagnetic field of Earth from being impacted by solar-origin magnetic elements, they are not completely impervious to their impact as was demonstrated during a solar eclipse [25]. Certainly disease risk is only one aspect of the impact of magnetic disturbances on humans, the other is on performance in space over the long term, or even more subtle influences on performance on Earth during the dynamics of the aging process.

Thus, summarizing the above discussion, we have learned considerably about human diversity and its impact on the variability in responses to LEO conditions, and we continue to learn more as more astronauts spend time in LEO conditions for longer periods of time. Thus, emerging findings related to skin health [26], and eye health [27] are being observed, and these are also associated with aging on Earth. In total, such findings as those addressed above has led some to speculate that living in space is a form of "accelerated aging" [28-30]. While we have learned some about human diversity in response to alterations in boundary condition parameters that likely impacted evolutionary pressures which have led to Homo sapiens, such responses have not been investigated in detail as the focus up to now have been discovering how to overcome these responses in order to alleviate risks to astronauts health and compromise of their performance. Using the responses to space flight and its associated alterations compared to living on Earth (e.g. microgravity, radiation exposure, magnetic field fluctuations ) to learn more about life on Earth and how such responses could be related to disease development and progression has not been a priority. And thus, this is somewhat of a missed opportunity to advance our understanding of health and risks to health on Earth [31]. Assessing the pattern of biologic and physiologic responses to space flight in an integrated manner, and correlating it with family history, genome sequencing, assessment of the dynamic epigenetic changes over time, and temporal relationships regarding the changes observed in a dynamic manner, could realize a wealth of information.

\section{ENHANCING THE “RETURN ON INVESTMENT” BY OPTIMIZING INFORMATION GAIN FROM ASTRONAUTS}

The world's space agencies spend a considerable amount of resources on the selection and training of astronauts. Most of them have multiple advanced degrees with highly developed skills, and they have had to pass a variety of physical exams. In addition, considerable effort has gone into development of countermeasures to minimize the impact of space conditions on human physiology and psychology, but to varying degrees of success (which is interesting and very telling in itself!). Only a subset of astronauts ac- 
tually get missions (single or multiple) into space, and for those that do get such missions, participation in experimental scientific protocols is largely voluntary and even those focused on astronaut physiology and psychology are restricted (e.g. genomic analysis and genetics cannot be used to prevent an astronaut from obtaining a space mission).

In part, the above scenario is constrained by the job description astronauts were hired under, and the fact they are considered "employees" of their respective space agencies. Once their time as an astronaut is completed, they retire to develop other careers. Many still retain health care and evaluation by medical experts, but mainly as former employees.

The above scenario has evolved with the development and progression of space agencies and their mandates (e.g. Apollo missions, going to the moon, Skylab, ISS, and now preparing for deep space missions). The question now is, are these relationships and constraints still appropriate, or should they be re-evaluated and re-defined to get a better return on the investment in astronauts and their time in space, as well as improve astronaut health while in space and after their astronaut career is over? In other words, should the job descriptions be changed, should participation in research be mandated, are astronauts just employees or should they be considered something else and for life, is it enough to just focus on astronaut health while planning for and participating in space missions? As "investors" in the space programs, should tax payers who will never get to space, expect more from the programs in relation to impact on humans on Earth and in return, invest more into the programs if the benefit is greater and more tangible? The answer is likely yes. In part, the return may be technological, but in the end a direct benefit to humans has to be shown. To do this will require not only space agency involvement, but also federal and other agencies focused on human health on earth to form partnerships which will be beneficial to both via more rapid and complete understanding of Homo sapiens, the adaptations that were required to exist on Earth and the risks to their health that are "buried" in their genomes because of that environment!

\section{SHOULD WE CONSIDER OTHER APPROACHES TO UTILIZE SPACE TO BETTER UNDERSTAND HUMAN HEALTH ON EARTH?}

If it is not possible to utilize astronauts to better understand risks to health on Earth as discussed above, are there other approaches one could envision? One approach, although expensive and potentially risky, would be to hire people to populate the ISS and be specifically recruited to be "space guinea pigs" for an extended period of time (6 - 12 months/trip). They would not have to be trained as current astronauts and run the ISS per se, but be tasked with specific experiments to be performed on site among themselves, and with other life science protocols. Thus, rather than abandon the ISS and let its orbit decay and burn up in the future, utilize it for specific gain to better understand who Homo sapiens are, and how our diversity contributes to disease risk across the lifespan on Earth using an altered environment (e.g. space). Thus, the ISS could become a "living laboratory" with an altered agenda from its initial goals. Some may argue that we could learn just as much from space analogues on Earth, but while these analogues are informative, they do not duplicate all aspects of LEO. An intriguing option, but certainly there would have to be some will to implement it and a careful risk/benefit analysis performed before embarking on such an endeavor!

One consideration to make it more successful is to take an integrated approach to understanding the impact of space flight on humans rather than the somewhat "fragmented" (e.g. body part focused, disease entity) approaches currently utilized. Such a fragmented approach may provide useful information, but unless integrated is not as useful as it could be. Implementation of more system biology, systems physiology approaches [32-34] would provide much more information that would facilitate translation back to Earth, as well as for translation to astronaut health. As most if not all physiologic systems are interdependent (e.g. for example: muscles affect bone integrity, cardiovascular health affects cognition and most other systems, the brain and vision systems affect mobility and navigation in the environment, neuroendocrine mediators affect multiple systems), unless the space-related changes are assessed in a temporal and integrated manner, it is not possible to accurately assess primary changes from secondary alterations. Thus, integration is central and essential via application of multidisciplinary approaches at the biological 
and physiological levels, as well as incorporation of advanced computer systems (e.g. IBM's Watson Project) and Artificial Intelligence (AI) perspectives.

\section{ACKNOWLEDGEMENTS}

The author thanks Drs. Richard Hughson, Nicole Buckley, Peter Suedfeld, Robert Thirsk, Nigel G. Shrive, Cyril B. Frank, and many others for the myriad of discussions over the past decades regarding the topic of this review. The author has been supported by funds from the Canadian Space Agency in the past, and acknowledges the current support from the AHS Strategic Clinical Networks Program.

\section{CONFLICTS OF INTEREST}

The author attests he has no conflict of interests to disclose.

\section{REFERENCES}

1. Darwin, C. (1859) The Origin of the Species.

2. Moller, A.P. and Moussesu, T.A. (2013) The Effects of Natural Variation in Background Radioactivity on Humans, Animals and Other Organisms. Biological Reviews of the Cambridge. Philosophical Society, 88, 226-254. https://doi.org/10.1111/j.1469-185X.2012.00249.x

3. Courtillot, V. and Le Mouel, J.L. (1988) Time Variations of the Earth's Magnetic Field: From Daily to Secular. Annual Review Earth Planetary Science, 16, 389-476. https://doi.org/10.1146/annurev.ea.16.050188.002133

4. Tattersall, I. (2009) Human Origins: Out of Africa. Proceeding of the National Academy of Sciences (USA), 106, 16018-16021. https://doi.org/10.1073/pnas.0903207106

5. Hood, L. and Galas, D. (2003) The Digital Code of DNA. Nature, 421, 444-448. https://doi.org/10.1038/nature01410

6. Ueno, S. (2012) Studies on Magnetism and Bioelectromagnetics for 45 Years: From Magnetic Analog Memory to Human Brain Stimulation and Imaging. Bioelectromagnetics, 33, 3-22. https://doi.org/10.1002/bem.20714

7. Ploutz-Snyder, L., Bloomfield, S., Smith, S.M., Hunter, S.K., Templeton, K. and Bemben, D. (2014) Effects of Sex and Gender on Adaptation to Space: Musculoskeletal Health. Journal of Women's Health, 23, 963-966.

https://doi.org/10.1089/jwh.2014.4910

8. Iwamoto, J., Takeda, T. and Sato, Y. (2005) Interventions to Prevent Bone Loss in Astronauts during Space Flight. Keio Journal of Medicine, 54, 55-59. https://doi.org/10.2302/kjm.54.55

9. Feinberg, A.P. (2018) The Key Role of Epigenetics on Human Disease Prevention and Mitigation. New England Journal of Medicine, 378, 1323-1334. https://doi.org/10.1056/NEJMra1402513

10. Hughes-Fulford, M., Chang, T.T., Martinez, E.M. and Li, C.F. (2015) Spaceflight Alters Expression of microRNA during T-Cell Activation. FASEB Journal, 29, 4893-4900. https://doi.org/10.1096/fj.15-277392

11. Fogel, O., Richard-Miceli, C. and Tost, J. (2017) Epigenetic Changes in Dhronic Inflammatory Diseases. $A d$ vances in Protein Chemistry and Structural Biology, 106, 139-189. https://doi.org/10.1016/bs.apcsb.2016.09.003

12. Hughson, R.L. (2009) Recent Findings in Cardiovascular Physiology with Space Travel. Respiratory Physiology and Neurobiology, 169, S38-S41. https://doi.org/10.1016/j.resp.2009.07.017

13. Hughson, R.L., Robertson, A.D., Arbeille, P., Shoemaker, J.K., Rush, J.W.E., Fraser, K.S. and Greaves, D.K. (2016) Increased Postflight Carotid Artery Stiffness and Inflight Insulin Resistance Resulting from 6-mo Spaceflight in Male and Female Astronauts. American Journal Physiology Heart Circulatory Physiology, 310, H628-H638. https://doi.org/10.1152/ajpheart.00802.2015

14. Wilson, D.M. $3^{\text {rd }}$, Kim, D., Berquist, B.R. and Sigurdson, A.J. (2011) Variation in Base Excision Repair Capacity. 
Mutation Research, 711, 100-112. https://doi.org/10.1016/j.mrfmmm.2010.12.004

15. Yatagai, F. and Ishioka, N. (2014) Are Biological Effects of Space Radiation Really Altered under the Microgravity Environment? Life Sciences in Space Research, 3, 76-89. https://doi.org/10.1016/j.1ssr.2014.09.005

16. Baillet, S. (2017) Magnetoencephalography for Brain Electrophysiology and Imaging. Nature Neuroscience, 20, 327-339. https://doi.org/10.1038/nn.4504

17. Delp, M.D., Charvat, J.M., Limolo, C.L., Globus, R.K. and Ghosh, P. (2016) Apollo Lunar Astronauts Show Higher Cardiovascular Disease Mortality: Possible Deep Space Radiation Effects on the Vascular Endothelium. Scientific Reports, 6, Article No. 29901.

18. Caswell, J.M., Singh, M. and Persinger, M.A. (2016) Simulated Sudden Increases in Geomagnetic Activity and Its Effect on Heart Rate Variability: Experimental Verification of Correlation Studies. Life Sciences in Space Research, 10, 47-52. https://doi.org/10.1016/j.lssr.2016.08.001

19. Cucinotta, F.A., Hamada, N. and Little, M.P. (2016) No Evidence for an Increase in Circulatory Disease Mortality in Astronauts Following Space Radiation Exposures. Life Sciences in Space Research, 10, 53-56.

https://doi.org/10.1016/j.lssr.2016.08.002

20. Baevsky, R.M, Petrov, V.M. and Chernikova, A.G. (1998) Regulation of Autonomic Nervous System in Space and Magnetic Storms. Advances in Space Research, 22, 227-234. https://doi.org/10.1016/S0273-1177(98)80014-8

21. Breus, T.K., Baevskii, R.M. and Chernikova, A.G. (2012) Effects of Geomagnetic Disturbances on Humans Functional State in Space Flight. Journal of Biomedical Science and Engineering, 5, 341-355.

https://doi.org/10.4236/jbise.2012.56044

22. Cucinotta, F.A., Alp, M., Sulzman, F.M. and Wang, M. (2014) Space Radiation Risks to the Central Nervous System. Life Sciences in Space Research, 2, 54-69. https://doi.org/10.1016/j.lssr.2014.06.003

23. Kay, R.W. (2004) Schizophrenia and Season of Birth: Relationship to Geomagnetic Storms. Schizophrenia Research, 66, 7-20. https://doi.org/10.1016/S0920-9964(02)00495-4

24. Mulligan, B.P. and Persinger, M.A. (2012) Experimental Simulation of the Effects of Sudden Increases in Geomagnetic Activity upon Quantitative Measures of Human Brain Activity: Validation of Correlational Studies. Neuroscience Letters, 516, 54-56. https://doi.org/10.1016/j.neulet.2012.03.054

25. Keshavan, M.S., Gangadhar, B.N., Gautam, R.U., Ajit, A. and Kapur, R.L. (1981) Convulsive Threshold in Humans and Rats and Magnetic Field Changes: Observations during Total Solar Eclipse. Neuroscience Letters, 22, 205-208. https://doi.org/10.1016/0304-3940(81)90089-6

26. Neutelings, T., Nusgens, B.V., Liu, Y., Tavella, S., Ruggiu, A., Cancedda, R., Gabriel, M., Colige, A. and Lambert, C. (2015) Skin Physiology in Microgravity: A 3-Month Stay Aboard ISS Induces Dermal Atrophy and Affects Cutaneous Muscle and Hair Follicles Cycling in Mice. Microgravity, 1, 15002.

https://doi.org/10.1038/npjmgrav.2015.2

27. Chylack, L.T. Jr., Feiveson, A.H., Peterson, L.E., Wear, M.L., Marak, L.J., Hardy, D.S., Chappell, L.J. and Cucinotta, F.A. (2012) NASCA Report 2: Longitudinal Study of Relationship of Exposure to Space Radiation and Risk of Lens Opacity. Radiation Research, 178, 25-32. https://doi.org/10.1667/RR2876.1

28. Ray, E.K. (1991) Introduction: Are Aging and Space Effects Similar? Experimental Gerontology, 26, 123-129. https://doi.org/10.1016/0531-5565(91)90002-4

29. Cristofalo, V.J. (1991) On Understanding the Biology of Aging: Studies in Space. Experimental Gerontology, 26, 137-138. https://doi.org/10.1016/0531-5565(91)90005-7

30. Vernikos, J. and Schneider, V.S. (2010) Space, Gravity and the Physiology of Aging: Parallel or Convergent Disciplines? A Mini-Review. Gerontology, 56, 157-166. https://doi.org/10.1159/000252852

31. Demontis, G.C., Germani, M.M., Caiani, E.G., Barravecchia, I., Passino, C. and Angeloni, D. (2017) Human 
Pathophysiological Adaptations to the Space Environment. Frontiers in Physiology, 8, 547. https://doi.org/10.3389/fphys.2017.00547

32. Ideker, T., Dutkowski, J. and Hood, L. (2011) Boosting Signal-to-Noise in Complex Biology: Prior Knowledge Is Power. Cell, 144, 860-863. https://doi.org/10.1016/j.cell.2011.03.007

33. Hood, L. and Tian, Q. (2012) Systems Approaches to Biology and Disease Enable Translational Systems Medicine. Genomics, Proteomics and Bioinformatics, 10, 181-185. https://doi.org/10.1016/j.gpb.2012.08.004

34. Trachana, K., Bargaje, R., Glusman, G., Price, N.D., Huang, S. and Hood, L.E. (2018) Takings Systems Medicine to Heart. Circulation Research, 122, 1276-1289. https://doi.org/10.1161/CIRCRESAHA.117.310999 